\title{
RECUPERAÇÃO DE COBRE DO LICOR DE MINÉRIO LIMONÍTICO ATRAVÉS DA TÉCNICA DE EXTRAÇÃO POR SOLVENTES *
}

\author{
Paula Aliprandini ${ }^{1}$ \\ Mónica Maria Jimenez Correa ${ }^{2}$ \\ Jorge Alberto Soares Tenório ${ }^{3}$ \\ Denise Crocce Romano Espinosa ${ }^{4}$
}

Resumo

O licor de lixiviação do minério limonítico tem como foco a recuperação dos metais níquel e cobalto. No entanto, o minério apresenta em sua composição outros metais como o cobre. Este metal não é o foco do beneficiamento, porém tem valor comercial, o que faz com que a recuperação desse metal agrege valor ao tratamento do minério. A técnica de extração por solventes é uma técnica conhecida na hidrometalurgia para recuperação de metais. Para a recuperação de cobre alguns extratantes são conhecidos, como os do tipo hidroximas. Pertencente a essa classe, o extratante Acorga M5640 é usado na extração do cobre. Esse trabalho buscou avaliar o pH de extração do cobre a partir de uma solução sintética baseada no licor de lixiviação do minério limonítico. Foi levada em consideração a solução após o pré-tratamento de remoção do ferro. Além disso, foram avaliadas diferentes concentrações do extratante Acorga M5640 na fase orgânica a fim de avaliar a quantidade necessária para a extração total do cobre sem que houvesse coextração dos demais metais. Foi possível extrair $99 \%$ do cobre em solução usando Acorga M5640 na concentração de 5\% em volume e pH de trabalho igual a 2 ..

Palavras-chave: Hidrometalurgia; Extração de cobre; Acorga M5640; Extração por solventes.

\section{COPPER RECOVERY OF LIMONITE ORE LIQUOR USING SOLVENT EXTRACTION TECHNIQUE}

\section{Abstract}

The leach liquor of limonite ore has focus on recovery metals as nickel and cobalt. However, the composition of the ore presents others metals, as copper. This metal is not recovered during the ore beneficiation, but it has commercial value. In addition, copper recovery can add value to ore treatment. Solvent extraction technique is used in hydrometallurgical process for the recovery of metals. Some extractants are known for copper recovery, such as the hydroximes type. Acorga M5640 is an extractant that belongs to the hydroximes class and it is used to extract copper. This study aimed to evaluate the $\mathrm{pH}$ of copper extraction from a synthetic solution based on leaching liquor of limonite ore. The solution was prepared considering pretreatment after the iron removal. Different concentrations of Acorga M5640 in the organic phase were studied. The extractant concentration indicated the amount required for the complete extraction of copper, without coextraction of the other metals. It was possible to extract $99 \%$ of the copper using Acorga M5640 in the concentration of $5 \%$ by volume and working $\mathrm{pH} 2$.

Keywords: Hydrometallurgy; Copper extraction; Acorga M5640; Solvent extraction.

1 Engenheira de Materiais, Mestre em Ciências, Doutoranda no Departamento de Engenharia Química, Universidade de São Paulo, São Paulo, São Paulo, Brasil.

2 Engenheira Química, mestre em ciências, Doutoranda do Departamento de Engenharia Química, Escola Politécnica da Universidade de São Paulo, São Paulo, São Paulo, Brasil.

3 Engenheiro Metalurgista, Doutor em engenharia metalúrgica, professor titular, Departamento de Engenharia Química, Escola Politécnica da Universidade de São Paulo, São Paulo, São Paulo, Brasil.

4 Engenheira metalurgista, doutora em engenharia metalúrgica, professora associada, Departamento de Engenharia Química, Escola Politécnica da Universidade de São Paulo, São Paulo, São Paulo, Brasil.. 


\section{INTRODUÇÃO}

O beneficiamento do minério limonítico com o fim de recuperar níquel e cobalto já é conhecido [1-4]. No entanto, além dos dois metais com interesse tradicional de recuperação, o minério apresenta composição complexa e variada de diferentes metais. Um dos metais que está presente no minério limonítico é o cobre $(\mathrm{Cu})$, que é um metal com interesse comercial. A presença de outros metais de interesse no minério agrega valor ao beneficiamento do minério. No minério limonítico a presença de cobre é em concentrações relativas baixas. No minério a presença de cobre varia entre $0,001 \%$ até $0,044 \%$ (em peso) [5-7].

O cobre é um metal estratégico para o abastecimento da indústria. A Figura 1 mostra um comparativo do metal com outros metais de interesse comercial. É possível observar que o $\mathrm{Cu}$ apresenta baixo risco de abastecimento quanto comparado aos demais metais listados. No entanto, quanto ao impacto da restrição de abastecimento, a classificação é de moderada para alta. Isso mostra a importância desse metal para a indústria [8].

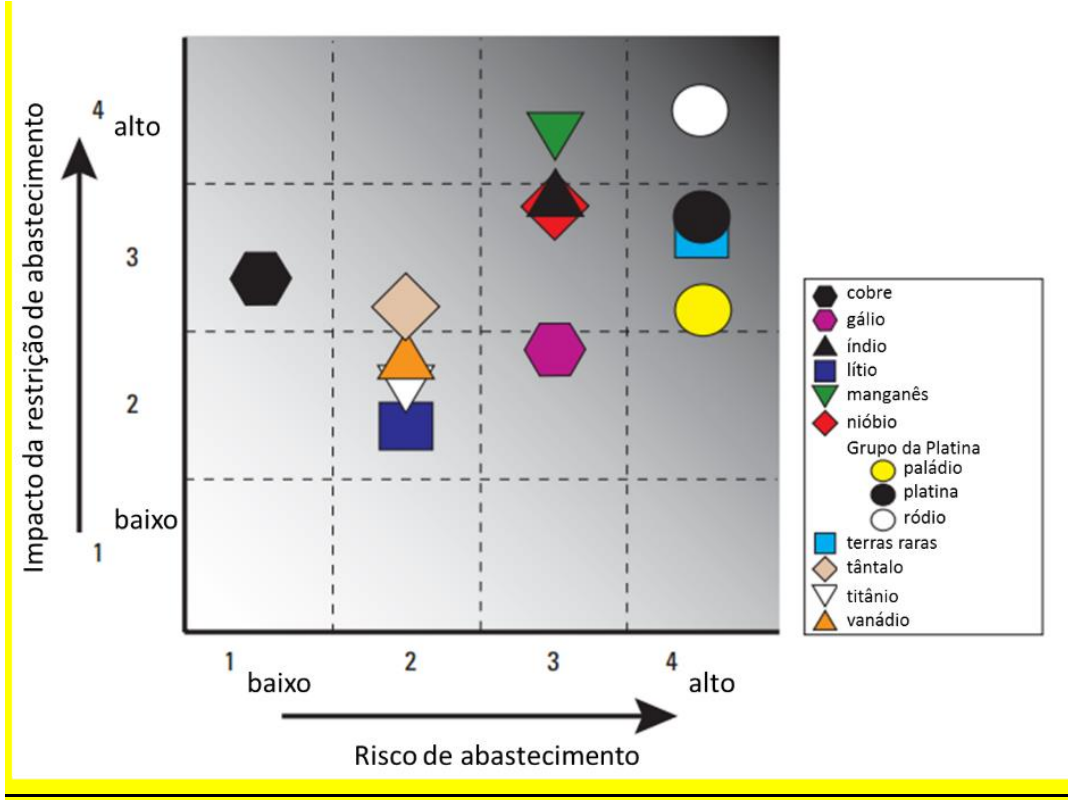

Figura 1. Relação entre o risco de abastecimento e o impacto de restrição de abastecimento para alguns metais considerados com importância econômica [8] (Adaptado).

A destinação do cobre é principalmente para a construção civil e equipamentos de transporte e eletroeletrônicos [9]. O minério limonítico não é a fonte tradicional de cobre, porém pode ser futuramente uma fonte alternativa de obtenção do minério.

O beneficiamento do minério limonítico pode ocorrer pode rotas hidrometalúrgicas [10]. Uma das técnicas utilizadas é a extração por solventes.

A extração por solventes é uma técnica já conhecida para a recuperação de metais e remoção de impurezas a partir de soluções lixiviadas de minérios. Trata-se de colocar em contato sob agitação duas soluções imiscíveis: uma fase orgânica e uma fase aquosa [2,11-15]. A fase aquosa é proveniente do lixiviado e a fase orgânica é formada pela junção de um extratante e um diluente. O primeiro é o principal reagente da fase e o responsável pela remoção seletiva dos íons metálicos presentes no lixiviado [2,13]. 
Para a extração do $\mathrm{Cu}$, alguns extratantes são utilizados. Os extratantes do tipo hidroximas são destinados para a extração do cobre e do níquel [16]. O extratante Acorga M5640, que pertence à família dos extratantes hidroxioxima, tem como foco a seletividade do cobre frente aos outros metais [17,18]. A Figura 2 mostra a estrutura química do composto 5-nonilsalicilaldoxima, que forma o extratante [18].<smiles></smiles>

Figura 2. Estrutura química do extratante Acorga M5640 [18].

A reação do extratante Acorga M5640 com o metal é do tipo quelante, ocorrendo liberação de hidrogênio durante a troca com o cobre na extração. De maneira geral, os extratantes quelantes atuam envolvendo o íon metálico por estruturas contendo anéis que são solúveis na fase orgânica [2]. A formação esférica entre o íon e as moléculas do extratante limita a extração a íons com tamanhos específicos (Figura $3)$. Isso torna esses extratantes seletivos para íons metálicos específicos [2,15]. A reação que representa a extração do cobre através de extratantes quelantes é a Equação 1 [19], sendo L o extratante.

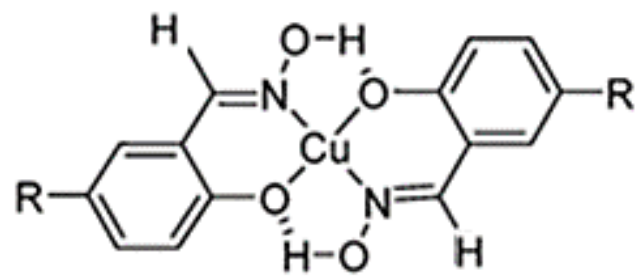

Figura 3. Representação das reações de extração que ocorrem durante a extração/reextração do íon metálico cobre em contato com o extratante Acorga M5640 [20] (Adaptado).

$$
2 \mathrm{LH}_{(\text {org })}+\mathrm{Cu}^{2+} \leftrightarrow \mathrm{CuL}_{2}(\text { org })+2 \mathrm{H}^{+}
$$

Esse trabalho tem como objetivo a avaliação do pH de extração do cobre e a concentração do extratante Acorga M5640 na fase orgânica para determinar as melhores condições para a extração do metal. Além disso, avaliar o comportamento dos demais presentes em solução durante as condições determinadas para a extração do cobre.

\section{MATERIAIS E MÉTODOS}

Para esse trabalho, uma solução sintética baseada no licor de lixiviação do minério limonítico foi preparada. Foi considerada a solução após o pré-tratamento para remoção do ferro da solução lixiviada. A Tabela 1 mostra a composição da solução 
sintética multielementar, bem como as concentrações usadas. Para a preparação da solução foram utilizados os metais na forma de sulfato.

Tabela 1. Concentração dos metais presentes na solução sintética baseada no licor de lixiviação do minério limonítico.

\begin{tabular}{ccccccccc}
\hline Metais & Al & Co & $\mathbf{C u}$ & $\mathbf{C r}$ & $\mathbf{~ N i}$ & $\mathbf{M g}$ & $\mathbf{M n}$ & $\mathbf{Z n}$ \\
\hline $\begin{array}{c}\text { Concentrações } \\
\mathbf{g} / \mathbf{L}\end{array}$ & 4,57 & 0,05 & 0,13 & 0,20 & 2,52 & 7,77 & 0,36 & 0,03 \\
\hline
\end{tabular}

Inicialmente foram avaliados diferentes valores de pH para a extração entre 2 e 3,5, sendo o intervalo igual a 0,5 . Como já mencionado anteriormente, o pH de início da solução foi ajustado para o pH 2. Isso se deve ao fato de que foi considerada a solução após o pré-tratamento da remoção do ferro [21].

No estudo do $\mathrm{pH}$ a solução orgânica foi preparada com a concentração do extratante Acorga M5640 igual a 20\% v/v diluído em querosene. A temperatura de ensaio foi de $25^{\circ} \mathrm{C}\left( \pm 2^{\circ} \mathrm{C}\right)$, com tempo de contato de 10 minutos e relação entre as fases aquosa e orgânica $(A / O)$ igual 1/1. Para o ajuste do $\mathrm{pH}$ nas diferentes etapas do trabalho, foi utilizada uma solução de hidróxido de sódio $(\mathrm{NaOH})$ na concentração $1 \mathrm{M}(\mathrm{mol} / \mathrm{L})$.

A segunda etapa do trabalho foi avaliar a concentração do extratante para a extração do metal no $\mathrm{pH}$ determinado na primeira etapa. Para isso a mesma relação entre as fases foi mantida $(A / O=1 / 1)$ e o tempo de contato mantido de 10 minutos. Cinco soluções orgânicas foram preparadas utilizando as concentrações de 1, 5, 10, 15 e $20 \%$ (v/v) do extratante Acorga M5640 no diluente querosene.

A determinação da concentração dos metais nas fases aquosa após a extração foi determinada pela técnica de Espectroscopia de Fluorescência de Raios X por Energia Dispersiva (EDX).

\section{RESULTADOS E DISCUSSÃO}

O primeiro estudo avaliou $\mathrm{o} \mathrm{pH}$ de extração do cobre presente na solução multielementar sintética. As porcentagens de extração dos metais durante a extração com Acorga M5640 na concentração $20 \% \mathrm{v} / \mathrm{v}$, relação entre as fases $A / O$ $1 / 1$ e para a faixa de pH entre 2 e 3,5 podem ser observadas na Figura 4.

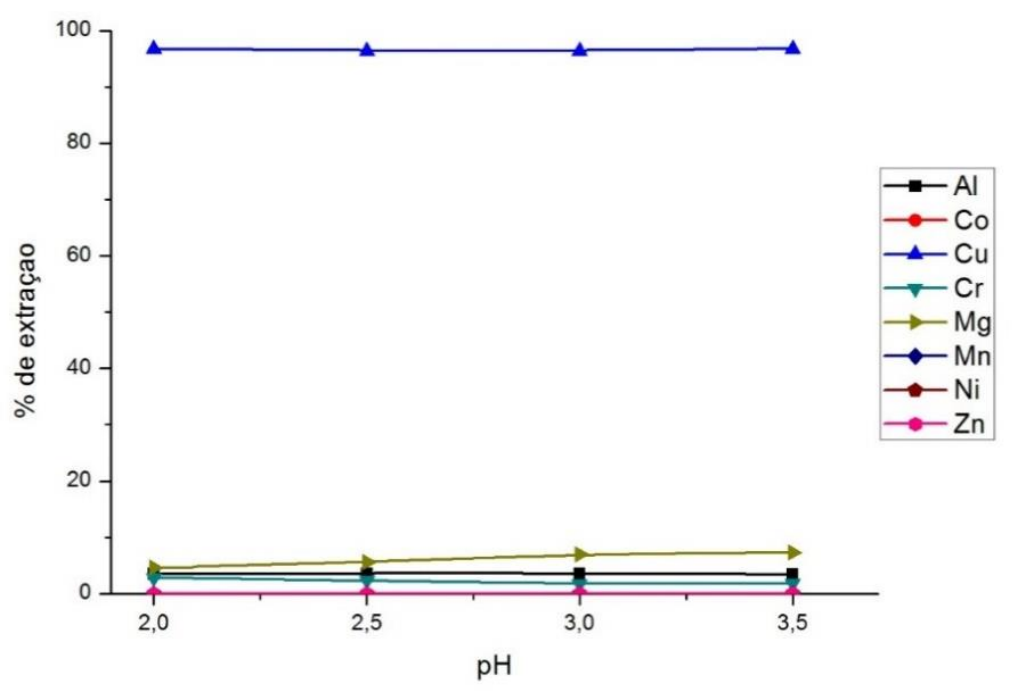

Figura 4. Relação da porcentagem de extração em cada valor de pH para cada metal presente em solução utilizando Acorga M5640 na concentração $20 \%$ v/v e relação $A / O$ de $1 / 1$. 
Foi possível observar a extração do cobre acima de $96 \%$ para a faixa de $\mathrm{pH}$ em estudo. Para os demais metais presentes na solução, apenas alumínio, cromo e magnésio apresentaram extração, no entanto, não superior a 7\%. A preferência pelo cobre mostrada nos resultados se devem à seletividade do metal para a extração utilizando o extratante Acorga M5640 [17].

Nessas condições avaliadas não houve extração do níquel, que também é considerado como de interesse pelo tipo de extratante utilizado [16].

O comportamento linear e constante para a extração do cobre nos valores de $\mathrm{pH}$ escolhidos mostra que a seletividade do extratante para com esse metal independe do $\mathrm{pH}$. A partir disso, foi escolhido o pH 2 para a extração do cobre. A variação do $\mathrm{pH}$ implicou em uso desnecessário do reagente básico, no caso desse estudo $\mathrm{NaOH}$, sem que houvesse alteração dos resultados obtidos.

A técnica de extração por solventes é baseada no cálculo de alguns parâmetros. É possível avaliar a seletividade do extratante entre um íon metálico em comparação com outro íon durante o processo de extração. $O$ fator de separação $S$ é um parâmetro comparativo entre a extração de dois metais nas mesmas condições. Ele é avaliado através da relação entre o coeficiente de distribuição $(D)$ do metal $x$ em relação ao $D$ do metal y (Equação 2) [2,12-14,22]. O coeficiente de distribuição, por sua vez, é a avaliação da tendência do metal ser extraído para a fase orgânica (Equação 3) $[2,12,22]$, sendo $[X]_{o}$ a concentração do metal $X$ na fase orgânica e $[X]_{a}$ a concentração do metal $X$ na fase aquosa.

$$
\begin{aligned}
& S=\frac{D_{x}}{D_{y}} \\
& D=\frac{[X] o}{[X] a}
\end{aligned}
$$

A Tabela 2 mostra o fator de separação entre o cobre e os metais que presentaram extração em pH 2, ou seja, alumínio, cromo e magnésio.

Tabela 2. Valores do fator de separação entre o cobre e os metais que foram extraídos utilizando Acorga M5640 (20\% v/v em querosene) em pH 2,0 e 25ㄷ.

\begin{tabular}{ccc}
\hline $\mathbf{S}_{\mathrm{Cu} / \mathrm{Al}}$ & $\mathrm{SCu}_{\mathrm{Cr}}$ & $\mathrm{S}_{\mathrm{Cu} / \mathrm{Mg}}$ \\
\hline 822,23 & 1000,93 & 746,81
\end{tabular}

O fator de separação entre o cobre e o cromo foi de 1000,93 , entre o cobre e 0 alumínio foi de 822,23 e entre o cobre e o magnésio foi de 746,81. Esses valores de fator de separação entre o cobre e os demais metais confirma a seletividade do extratante para a extração do cobre em relação aos demais metais presentes no licor.

A partir da escolha do $\mathrm{pH} 2$ de trabalho para a extração do cobre, foram avaliadas as diferentes concentrações em volume do extratante Acorga M5640 contidas na fase orgânica. A Figura 5 mostra o comportamento de extração dos metais para as concentrações de 1, 5, 10, 15 e 20\% (v/v) do extratante na fase orgânica. 


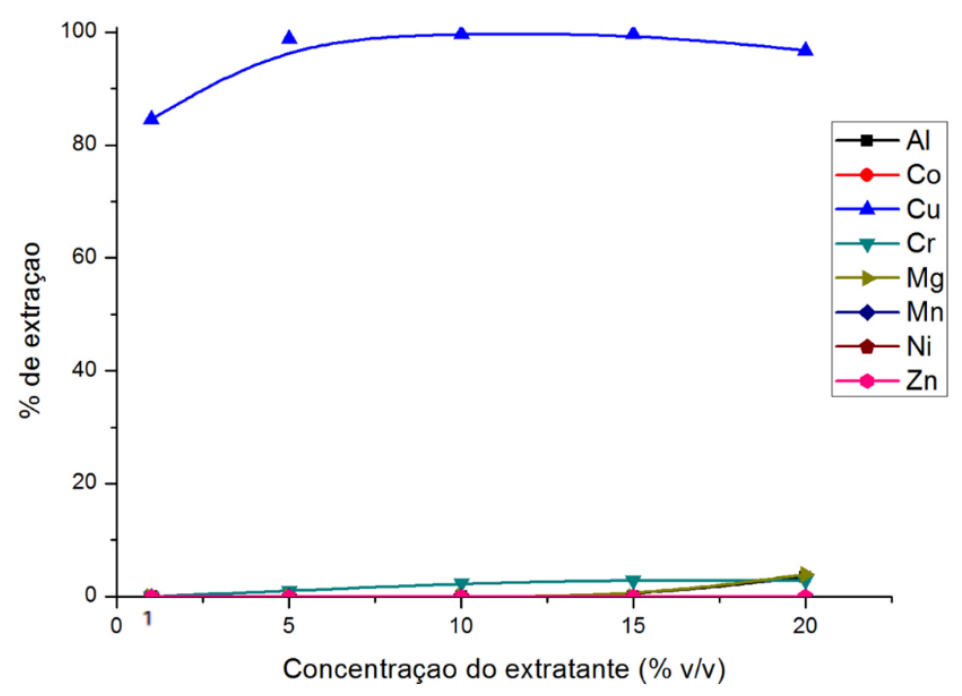

Figura 5. Porcentagem de extração dos metais em função da concentração do extratante Acorga M5640 (v/v) em querosene no pH 2, relação $A / O$ igual a 1/1 e 25ํ․

A concentração de $20 \%$ (v/v) de extratante, adotado na avaliação do pH de extração foi superior à concentração necessária para extrair todo o cobre presente no licor. Como pode ser observado na Figura 5, a porcentagem de extração do cobre foi de $85 \%$ em concentração de 1\% em volume de Acorga M5640 em querosene e de $99 \%$ quando $5 \%(\mathrm{v} / \mathrm{v})$ do extratante foi adicionado na fase orgânica. Os demais metais não apresentaram extração quando a solução aquosa foi contatada com a fase orgânica contendo 1\% (v/v) de extratante. Em concentração de 5\% em volume do extratante, houve apenas extração de cromo, porém em porcentagem inferior a $1 \%$. Ao longo do aumento da concentração do extratante houve aumento da extração de cromo chegando a 2,8\% em concentração do extratante igual a $20 \% \mathrm{v} / \mathrm{v}$. O alumínio e o magnésio apenas apresentaram extração em concentração igual a $20 \% \mathrm{v} / \mathrm{v}$ de Acorga M5640 na fase orgânica sendo, respectivamente, 3,5\% e 4,6\%.

Sendo assim, a concentração de 5\% em volume de Acorga M5640 na fase orgânica é suficiente para a extração de cobre em pH 2, relação $A / O$ igual a $1 / 1$ e temperatura de $25^{\circ} \mathrm{C}$.

O fator de separação entre o cobre e o cromo para a concentração de Acorga M5640 igual a $5 \%$ em volume é 8484,20 , enquanto que na concentração $20 \%$ o fator de separação é 1000,93. Isso representa aumento da separação dos metais em aproximadamente 8,5 vezes com a redução da quantidade de Acorga M5640 na fase orgânica.

\section{CONCLUSÃO}

Ao final do trabalho foi possível concluir que:

- A extração do cromo usando Acorga M5640 foi acima de 96\% em toda a faixa de $\mathrm{pH}$ estudada, indicando que o extratante é seletivo para o metal.

- Outros metais apresentaram extração nos pHs estudados, mas em porcentagem abaixo de $7 \%$.

- Não houve extração de níquel, o que poderia acontecer devido a classe do extrante também ser usado para a extração do níquel.

- A concentração de $20 \%$ em volume do extratante foi superior a necessária para a extração do cobre presente na solução estudada. 
- Foi possível extrair o cobre através do extratante Acorga M5640 na concentração $5 \% \mathrm{v} / \mathrm{v}$ em querosene, $\mathrm{pH}$ de equilíbrio 2 , relação $A / O 1 / 1$.

\section{Agradecimentos}

À Coordenação de Aperfeiçoamento de Pessoal de Nível Superior (CAPES) pelo suporte financeiro através da bolsa de doutorado.

Ao Conselho Nacional de Desenvolvimento Científico e Tecnológico (CNPq) pelo suporte financeiro através da bolsa de doutorado.

À Fundação de Amparo à Pesquisa do Estado de São Paulo (FAPESP) pelo suporte financeiro através do projeto de pesquisa 2012/51871-9.

\section{REFERÊNCIAS}

$1 \quad$ Kyle J. Nickel laterite processing technologies - where to next? In: ALTA 2010 Nickel/Cobalt/Copper Conference; 2010. p. 1-36.

2 Gupta CK. Chemical Metallurgy Principles and Practice. Weinheim; 2003.

3 Neudorf D. Method for Nickel and Cobalt Recovery from Laterite Ores by Reaction with Concentrated Acid and Water Leaching. US 2006/0002835 A1, 2006.

4 Chang Y, Zhai X, Li B, Fu Y. Removal of iron from acidic leach liquor of lateritic nickel ore by goethite precipitate. Hydrometallurgy. 2010;101(1-2):84-7.

5 Tang JA, Valix M. Leaching of low grade limonite and nontronite ores by fungi metabolic acids. Miner Eng. 2006;19(12):1274-9.

6 Wang K, Li J, McDonald RG, Browner RE. Characterisation of iron-rich precipitates from synthetic atmospheric nickel laterite leach solutions. Miner Eng. 2013;40:1-11.

7 Basturkcu H, Acarkan N, Gock E. The role of mechanical activation on atmospheric leaching of a lateritic nickel ore. Int $\mathrm{J}$ ofMineral Process. 2017;163:1-8.

8 Long KR, Van Gosen BS, Foley NK, Cordier D. The Principal Rare Earth Elements Deposits of the United States: A Summary of Domestic Deposits and a Global Perspective. U.S. Geological Survey Scientific Investigations Report 2010-5220. 2012 [acesso em 18 maio 2017] Disponível em: http://pubs.usgs.gov/sir/2010/5220/

9 U.S.Geological Survey. Copper. In: Mineral commodity Summaries 2017: US Geological Survey. 2017. p. 202.

10 Guo X, Li D, Park KH, Tian Q, Wu Z. Leaching behavior of metals from a limonitic nickel laterite using a sulfation-roasting-leaching process. Hydrometallurgy. 2009;99:144-50.

11 Morais CA de, Albuquerque RO de, Ladeira ACQ. Processos Físicos e Químicos Utilizados na Indústria Mineral. Cad Temáticos Química Nov na Esc. 2014;8:9-17.

12 Habashi F. Principles of Extractive Metallurgy. New York: Gordon and Brach Science Publishers; 1970.

13 Kislik VS. Solvent Extraction: Classical and Novel Approaches. Elsevier. Elsevier; 2012.

14 Vogel Al. Análise Química Quantitativa. São Paulo: DTC Editora; 1992.

15 Free M. Hydrometallurgy: fundamentals and applications. John Wiley \& Sons; 2013. 
16 Flett DS. Solvent extraction in hydrometallurgy: the role of organophosphorus extractants. J Organomet Chem 2005;690(10):2426-2438.

17 Cytec. Acorga M5640 metal extraction reagent. 2011 [acesso em 25 maio 2017] Disponível em:

https://www.cytec.com/sites/default/files/datasheets/Acorga\%20M5640\%20Prod uct\%20Data\%20Sheet.pdf

18 Cytec. Mining Chemicals Handbook. Nova Jersey: Cytec Industries Inc.;2010.

19 Deep A, Kumar P, Carvalho JMR. Recovery of copper from zinc leaching liquor using ACORGA M5640. Sep Purif Technol. 2010;76(1):21-5.

20 Wilson AM, Bailey PJ, Tasker PA, Turkington JR, Grant RA, Love JB. Solvent extraction: the coordination chemistry behind extractive metallurgy. Chem Soc Rev. 2014;43(1):123-34.

21 Aliprandini P, Jiménez Correa MM, Tenório JAS, Espinosa DCR. Remoção de ferro da solução sintética de minério limonítico de níquel utilizando extração por solventes. In: ABM Week 2016. Rio de Janeiro; 2016.

22 Ritcey GM, Ashbrook AW. Solvent Extraction: principles and applications to process metallurgy - Vol1. - Parte1. Elsevier; 1984. 